\title{
Ecocardiografia de equinos Puro Sangue Árabe após exercício de enduro de diferentes intensidades
}

\author{
Echocardiography in Arabian horses after endurance exercise of different intensities
}

\author{
Camila Alfaro de Oliveira Bello ${ }^{I^{*}}$ Carlos Eduardo da Silva Vasconcelos ${ }^{\mathrm{I}}$ Roberta Ferro de Godoy

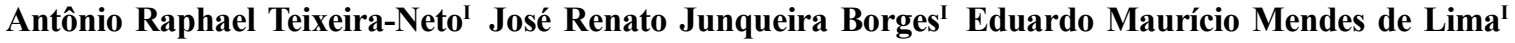

\section{RESUMO}

A ecocardiografia é um método não invasivo e adequado para a avaliação das alterações cardíacas em equinos, identificando assim aqueles animais que apresentaram melhor adaptação e condicionamento ao exercício a que foram submetidos. O objetivo deste estudo foi verificar por meio de exames ecocardiográficos em modo- $M$ e bidimensional os indices funcionais cardiacos de 15 equinos Puro Sangue Árabe, sendo nove machos e seis fêmeas, com idade variando de seis a 12 anos, submetidos a diferentes intensidades de exercício prolongado de enduro. Para tanto, foram divididos em três grupos, sendo que quatro animais percorreram $30 \mathrm{~km}$ (GI), seis animais percorreram $90 \mathrm{~km}$ (GII) e cinco animais percorreram $120 \mathrm{~km}$ (GIII). As avaliações ocorreram antes do início de cada prova, sendo considerado o momento zero $\left(M_{0}\right)$ e 30 minutos após percorrerem o último anel da correspondente prova, caracterizando o momento final (M). Diante do tratamento estatístico, não se verificaram diferenças significantes entre os indices obtidos no $M_{0}$ e $M_{f}$ do GI. De outra forma, no GII para VS, FC, VEs e VEd, assim como no GIII para VS, FC, VEs, VEd e SIVd, observou-se diferença estatística. Portanto, em equinos de enduro a ecocardiografia revelou que o exercício físico permitiu o estabelecimento de indices funcionais cardiacos próprios, de acordo com a intensidade do exercício imposto.

Palavras-chave: enduro, ecocardiografia, equinos.

\section{ABSTRACT}

Echocardiography is a noninvasive and accurate evaluation of cardiac abnormalities in horses, thereby determining those animals that show better adaptation to exercise and conditioning to which they were submitted. The aim of this study was to verify by echocardiographic M-mode and two-dimensional cardiac functional indices of 15 purebred Arabian horses, nine males and six females, ranging in age from 6 to 12 years, subjected to different intensities prolonged endurance exercise. Thus, both groups were divided into three, in which four animals traveled $30 \mathrm{~km}$ (GI), six animals traveled $90 \mathrm{~km}$ (GII) and five animals traveled $120 \mathrm{~km}$ (GIII). Assessments occurred before the start of each test and was considered the time zero $\left(M_{0}\right)$; 30 minutes after running through the last ring of the race characterized the final moment $\left(M_{f}\right)$. Before the treatment there were no statistically significant differences between the indexes obtained in the $M_{0}$ and $M_{f}$ of GI. Otherwise, it was observed statistical difference in GII for stroke volume, heart rate, left ventricular internal diameter in diastole and systole, and in GIII for stroke volume, heart rate, left ventricular internal diameter in diastole and systole and interventricular septal thickness in diastole. So endurance horses echocardiography revealed that the exercise enabled the assessment of cardiac functional indexes according to the intensity of the exercise tax.

Key words: endurance, echocardiography, horse.

\section{INTRODUÇÃO}

Existem diversos estudos realizados em humanos (WELSH et al.,2005; NEILAN et al., 2006) que executam exercício prolongado, revelando evidências ecocardiográficas de disfunção cardíaca, além de mostrarem que esse risco é maior em atletas com baixo nível de treinamento. Assim, para diversos autores, o exercício prolongado extenuante tem sido associado com disfunção transitória do ventrículo esquerdo e foi

IFaculdade de Agronomia e Medicina Veterinária, Universidade de Brasília (UnB), Instituto Central de Ciências, Ala Sul, Campus Universitário Darcy Ribeiro, CP 4508, 70760-701, Brasília, DF, Brasil. E-mail: mylabello@hotmail.com. *Autor para correspondência. 
denominado "fadiga cardíaca" (DOUGLAS et al., 1987; WELSH et al.,2005).

Nesse aspecto, embora estudos como o de LA GERCHE et al. (2004), que acompanharam atletas humanos de ultra-maratona, relatem que o exercício aeróbico prolongado não causou injúria miocárdica nem alterações significantes da função cardíaca pósexercício, outros autores como DÁVILA-ROMÁN et al. (1997), RIFAI et al. (1999) e TRIVAX et al. (2010) citam que o exercício extenuante prolongado pode levar a uma lesão miocárdica transitória mínima, sendo que o estresse cardíaco causado pelo exercício pode influenciar a função cardíaca.

Nas competições de enduro, em categorias de velocidade livre, os equinos podem percorrer até $160 \mathrm{~km}$ em um dia, resultando em um esforço físico muito grande. Como o exercício prolongado aumenta a carga de trabalho cardíaco, ele pode também causar algum grau de lesão miocárdica em cavalos e os comprometimentos metabólicos podem agravar o quadro (MICHIMA, 2007). Em especial, verifica-se que existem poucos trabalhos específicos sobre equinos de enduro que são acometidos de injúrias cardíacas provocadas pelo exercício físico (MICHIMA et al., 2003).

De acordo com os relatos acima, o objetivo deste estudo foi o de avaliar as alterações ecocardiográficas de equinos após terem sido submetidos a exercício prolongado de enduro de intensidade variável.

\section{MATERIAL E MÉTODOS}

Foram avaliados por meio da ecocardiografia 15 equinos da raça Puro Sangue Árabe submetidos a provas de enduro, sendo nove machos e seis fêmeas, com idade variando de seis a 12 anos, 311 a $486 \mathrm{~kg}$ de peso vivo, com, no mínimo, um ano de treinamento para enduro. Os animais foram divididos em três grupos, sendo que quatro destes percorreram $30 \mathrm{~km}$, compondo o grupo I (GI), seis animais percorreram $90 \mathrm{~km}$ formando o grupo II (GII) e cinco animais que percorreram $120 \mathrm{~km}$ formaram o grupo III (GIII). As avaliações ecocardiográficas ocorreram antes do início de cada prova, sendo considerado o momento zero $\left(\mathrm{M}_{0}\right)$ e 30 minutos após percorrerem o último anel da correspondente prova definindo o momento final $\left(\mathrm{M}_{\mathrm{f}}\right)$.

O exame ecocardiográfico foi realizado com aparelho de ecocardiografia (Sonosite Elite Plus), com transdutor convexo banda larga de 2 a $4 \mathrm{MHz}, 2 \mathrm{com}$ alcance de $25 \mathrm{~cm}$ de profundidade. Procedeu-se a avaliação no modo-M pela janela paraesternal direita, segundo as orientações da Sociedade Americana de Ecocardiografia (REEF, 1990).
O exame iniciou após a aplicação do gel ultrassonográfico com abordagem da janela paraesternal direita. As medidas de diâmetro interno do ventrículo esquerdo em sístole (VEs) e diástole (VEd), a espessura do septo interventricular em sístole (SIVs) e diástole (SIVd), a espessura da parede livre do ventrículo esquerdo em sístole (PLVEs) e em diástole (PLVEd) foram obtidas a partir da imagem das quatro câmaras, rotacionando o transdutor cerca de $90^{\circ}$, em sentido horário, obtendo assim uma imagem em plano transversal. $\mathrm{O}$ cursor em modo $\mathrm{M}$ foi posicionado visando à obtenção da imagem que determina a espessura do septo interventricular (SIV), diâmetro interno do ventrículo esquerdo (VE) e espessura da parede livre do ventrículo esquerdo (PLVE).

A fração de encurtamento (FS\%) foi calculada a partir dos valores obtidos do VEd e VEs através da fórmula: $\mathrm{FS} \%=[(\mathrm{VEd}-\mathrm{VEs}) / \mathrm{VEd}] \mathrm{x} 100$. A determinação do volume sistólico (VS) foi feita pela fórmula modificada de Teicholz: [7x(VE) $\left.)^{3} / 2,4+\mathrm{VE}\right]$ (TEICHOLZ et al., 1976). Por fim, o débito cardíaco (DC) foi obtido multiplicando-se o VS pela frequência cardíaca (FC).

Os índices obtidos nos dois momentos para cada um dos grupos passaram pelo teste de normalidade de Kolmogorov-Smirnov e, em seguida, foram submetidos ao tratamento estatístico por meio da aplicação do Teste "t" de Student, com nível de significância de $5 \%$.

\section{RESULTADOS E DISCUSSÃO}

Os resultados encontrados tiveram como base o tratamento estatístico isolado de cada índice entre o repouso $\left(M_{0}\right)$ e após o exercício $\left(M_{f}\right)$ para cada um dos três grupos (GI, GII e GIII) separadamente (Tabela 1).

Em todos os grupos, verificou-se diminuição do diâmetro interno do ventrículo esquerdo, em sístole e diástole, em relação aos dois momentos avaliados. Sobretudo, encontrou-se diferença estatisticamente significativa apenas entre os valores obtidos ecocardiograficamente em GII e GIII. Essa constatação vem confirmar o estudo de MARR et al. (1999), quando demonstraram diminuição significante do diâmetro do ventrículo esquerdo em sístole e diástole, em equinos treinados em esteira rolante. Da mesma forma, esse resultado foi observado para cavalos de enduro, conforme BERTONE et al. (1987), e humanos submetidos a exercício prolongado, de acordo com YAMAZAKI et al. (1990). Para NAYLOR et al. (1993), a redução do volume de sangue total, relacionado à desidratação ou uma maior redistribuição de sangue 
Tabela 1 - Representação dos índices funcionais cardíacos de equinos em repouso $\left(\mathrm{M}_{0}\right)$ e após exercício $\left(\mathrm{M}_{\mathrm{f}}\right)$ prolongado de enduro de diferentes intensidades (média+desvio padrão).

\begin{tabular}{|c|c|c|c|c|c|c|}
\hline & \multicolumn{2}{|c|}{--------------------'GI--------------------- } & \multicolumn{2}{|c|}{--------------------GII-------------------- } & \multicolumn{2}{|c|}{------------------GIII------------------ } \\
\hline & $\mathrm{M}_{0}$ & $\mathrm{M}_{\mathrm{f}}$ & $\mathrm{M}_{0}$ & $\mathrm{M}_{\mathrm{f}}$ & $\mathrm{M}_{0}$ & $\mathrm{M}_{\mathrm{f}}$ \\
\hline SIVd (cm) & $3,00 \pm 0,54$ & $3,14 \pm 0,72$ & $3,08 \pm 0,24$ & $3,74 \pm 0,61$ & $3,15 \pm 0,21 *$ & $3,38 \pm 0,17^{*}$ \\
\hline SIVs (cm) & $4,31 \pm 0,93$ & $4,50 \pm 0,46$ & $4,70 \pm 0,26$ & $4,65 \pm 0,34$ & $4,75 \pm 0,46$ & $4,75 \pm 0,59$ \\
\hline VEd $(\mathrm{cm})$ & $11,42 \pm 0,67$ & $10,75 \pm 1,24$ & $11,12 \pm 1,11^{*}$ & $9,43 \pm 1,37^{*}$ & $11,84 \pm 0,76^{*}$ & $9,32 \pm 0,46^{*}$ \\
\hline VEs $(\mathrm{cm})$ & $7,36 \pm 0,69$ & $6,74 \pm 0,78$ & $7,23 \pm 1,14^{*}$ & $6,29 \pm 0,57^{*}$ & $7,54 \pm 0,50^{*}$ & $5,75 \pm 0,56^{*}$ \\
\hline PLVEd (cm) & $2,30 \pm 0,51$ & $2,13 \pm 0,05$ & $2,45 \pm 0,18$ & $2,66 \pm 0,25$ & $2,38 \pm 0,23$ & $2,44 \pm 0,19$ \\
\hline PLVEs (cm) & $3,47 \pm 1,00$ & $3,26 \pm 0,30$ & $3,58 \pm 0,59$ & $3,72 \pm 0,40$ & $3,7 \pm 0,79$ & $3,81 \pm 0,44$ \\
\hline FE $(\%)$ & $35,34 \pm 7,86$ & $37,17 \pm 4,22$ & $35,23 \pm 5,22$ & $32,75 \pm 6,02$ & $36,25 \pm 4,23$ & $38,34 \pm 5,09$ \\
\hline VS (ml) & $291,25 \pm 62,49$ & $238,45 \pm 61,97$ & $280,81 \pm 92,72 *$ & $201,63 \pm 41,84^{*}$ & $302,39 \pm 41,85^{*}$ & $165,64 \pm 35,76^{*}$ \\
\hline $\mathrm{FC}(\mathrm{bpm})$ & $34,00 \pm 2,83$ & $44,50 \pm 7,72$ & $41,2 \pm 5,76^{*}$ & $51,4 \pm 4,98^{*}$ & $34,8 \pm 6,26^{*}$ & $54,8 \pm 7,40^{*}$ \\
\hline $\mathrm{DC}(1 / \mathrm{min})$ & $9,91 \pm 2,41$ & $10,44 \pm 2,64$ & $11,17 \pm 2,57$ & $10,35 \pm 2,22$ & $10,42 \pm 1,77$ & $9,01 \pm 2,08$ \\
\hline
\end{tabular}

Valores com sobrescritos* em uma mesma linha são estatisticamente diferentes. Espessura do septo interventricular em diástole (SIVd); espessura do septo interventricular em sístole (SIVs); diâmetro interno do ventrículo esquerdo em diástole (VEd); diâmetro interno do ventrículo esquerdo em sístole (VEs); espessura da parede livre do ventrículo esquerdo em diástole (PLVEd); espessura da parede livre do ventrículo esquerdo em sístole (PLVEs); fração de encurtamento (FE); volume sistólico (VS); frequência cardíaca (FC); débito cardíaco (DC). Grupo de quatro animais que percorreram $30 \mathrm{~km}(\mathrm{GI})$; grupo de seis animais que percorreram $60 \mathrm{~km}$ (GII); grupo de cinco animais que percorreram $120 \mathrm{~km}$ (GIII).

para fora da cavidade torácica e para dentro da rede vascular da musculatura esquelética e pele foram fatores importantes que promoveram a redução do diâmetro interno do ventrículo esquerdo após exercício.

Em relação à espessura do septo interventricular, em diástole, foi possível observar que houve aumento dessa medida em todos os grupos e que, em sístole, esse achado foi variado conforme expresso na tabela 1 . Sobretudo, apenas para os animais do grupo GIII, verificou-se a ocorrência de diferença estatisticamente significante no que diz respeito à espessura do septo interventricular em diástole, o que corrobora o estudo de BERTONE et al. (1987), que avaliaram cavalos após exercício de baixa intensidade, aeróbico e prolongado e encontraram redução significante na espessura do septo interventricular. De forma semelhante, em um estudo mais recente, SCHEFER et al. (2010) observaram o aumento desse índice em equinos, após exercício em esteira com FC de $80 \mathrm{bpm}$. Entretanto, esse resultado não apresentou diferença estatística .

A espessura da parede livre do ventrículo esquerdo, tanto em sístole quanto em diástole no GI sofreu uma diminuição, diferindo do ocorrido em GII e GIII, em que a espessura sofreu um aumento, em sístole e diástole. Esses dados, porém, não apresentaram diferença estatística entre si. No que diz respeito à alteração de espessura de parede miocárdica após exercício, assim como em nosso estudo, os achados foram variados entre diversos autores (BERTONE et al.,1987; DOUGLAS et al.,1987; GEHLEN et al., 2005;
MICHIMA, 2007), porém essa alteração foi reversível (BERTONE etal.,1987; DOUGLAS etal.,1987; GEHLEN et al., 2005; MICHIMA, 2007). Portanto, esse dado isolado ainda não nos permitiu compreender qual a real importância da alteração deste índice.

Em todos os grupos, evidenciou-se diminuição do volume sistólico após o exercício, apresentando diferença estatisticamente significativa apenas nos equinos dos grupos GII e GIII. Esse achado coincidiu com DÁVILA-ROMAN et al. (1997), que observaram sinais de fadiga pelo esforço nos animais, quando ocorreu aumento na função ventricular esquerda global no período imediatamente após o exercício, refletindo na redução de volume sistólico e diastólico. Da mesma forma, KALLIOKOSKI et al. (2004) consideraram que alterações leves nos parâmetros de função ventricular esquerda, incluindo a redução do volume sistólico, foram sinais característicos de fadiga cardíaca em maratonistas humanos.

Para os animais do GI, foi verificado o aumento do DC, concordando com o proposto por MARR et al. (1999), quando afirmou que o débito cardíaco mostrou-se dependente da pré-carga e sua elevação foi observada em situações associadas ao maior retorno venoso e menor resistência periférica, como observado durante o exercício. Para POOLE \& ERICKSON (2008), esse aumento deve-se ainda à combinação com a policitemia induzida pelo baço e ao incremento da $\mathrm{FC}$, com uma menor contribuição do volume sistólico elevado. 
De outra forma, observou-se, no presente estudo, redução do DC no GII e GIII, com diferença estatística entre estes. Nesse sentido, há concordância com o proposto por NAYLOR et al. (1993), que observaram que, em cavalos desidratados, o débito cardíaco diminuiu durante o exercício. De acordo com SWENSON \& REECE (1999), isso foi atribuído a um deslocamento de fluido do compartimento intravascular para o extravascular, como um resultado da sudorese, acrescido ainda da vasodilatação periférica para facilitar a dissipação de calor. Dessa forma, mudanças e perda de fluidos podem resultar em redução do volume sanguíneo (DURANDO et al., 2002). Foi possível sugerir que a diminuição do débito cardíaco, nos animais dos grupos GII e GIII, esteve diretamente relacionada ao fato de esses animais terem percorrido percursos maiores, consequentemente, estiveram mais propensos à desidratação e exaustão, bem como estiveram sujeitos a maiores efeitos termorregulatórios.

A FE\% pode ser afetada por três fatores com maior frequência, são eles: a pré-carga, pós-carga e contratilidade, de forma individual ou em conjunto. Quando um valor baixo de FE\% é obtido, pode ser secundária a baixa pré-carga, alta pós-carga ou contratilidade reduzida (BOON, 1998), como o observado no GII de forma não significativa. Essa informação confirma os estudos de MARR et al. (1999), que não observaram redução significante da $\mathrm{FE} \%$ após exercício em esteira, porém ela foi mais marcante que em condições quentes e úmidas a frias, sugerindo que, sob essa condição, a função ventricular esquerda pode diminuir. De outra forma, esse achado foi distinto para o GI e GIII, observando assim o aumento desse índice. Nesse sentido, esses padrões distintos não apresentaram diferença estatística significante em nenhum dos grupos, sugerindo que a redução da FE\% foi uma condição variável e não significante após o exercício prolongado.

NAYLOR et al. (1993) afirmaram que a desidratação levou à recuperação mais lenta dos batimentos cardíacos. Da mesma forma, para ROSE (1983), cavalos de enduro com a frequência cardíaca maior do que 65-70bpm mostram-se mais susceptíveis a sofrerem desidratação e exaustão. Ainda FLAMÍNIO \& RUSH (1998) afirmaram que o período de recuperação prolongado indicou um condicionamento inadequado, desequilíbrio hidroeletrolítico ou dor. Avaliando o resultado supracitado, pode-se verificar que os animais deste estudo encontravam-se bem condicionados. Observou-se diferença estatística entre GII e GIII, provavelmente devido ao fato de esses grupos terem realizados exercícios mais intensos, favorecendo a ocorrência de maior desidratação e exaustão que os animais de GI, sem, entretanto, sair dos valores da normalidade.

\section{CONCLUSÃO}

Diante das respostas encontradas, pôde-se concluir que, nos equinos avaliados, a ecocardiografia foi eficiente na determinação dos índices funcionais cardíacos adquiridos após exercício de enduro de intensidade variada. Assim, os valores observados neste estudo não apresentaram diferença estatística para os animais que percorreram $30 \mathrm{~km}$. De outra forma, verificou-se a ocorrência de diferença estatística para os animais que percorreram $90 \mathrm{~km}$, em relação aos valores obtidos do VEs, VEd, FC e VS, e para os que percorreram $120 \mathrm{~km}$, em que foi encontrada diferença estatística em relação ao VEs, VEd, FC, VS e SIVd. Esses resultados sugerem que as alterações estiveram diretamente relacionadas à intensidade do exercício de enduro, podendo ainda estabelecer relação concomitantemente com a desidratação, pois se entende que, nos animais que percorreram maiores distâncias, essa condição tornou-se mais acentuada. Dessa forma, seria indicado o estabelecimento de parâmetros para cada um dos grupos que quantificasse a desidratação para melhor subsidiar estes achados.

\section{COMITÊ DE ÉTICA}

O trabalho recebeu parecer favorável para execução de acordo com o Comitê de Ética no uso animal do instituto de ciências biológicas da Universidade de Brasília, sob protocolo $111913 / 2009$.

\section{REFERÊNCIAS}

BERTONE, J.J. et al. M-mode echocardiographs of endurance horses in the recovery phase of long-distance competition. American Journal of Veterinary Research, v.48, p.17081712, 1987. Disponível em: <http://www.ncbi.nlm.nih.gov/ pubmed/3434918>. Acesso em: 20 out. 2010. doi: 3434918 .

BOON, J.A. Evaluation of size, function and hemodynamics. In: _____. Manual of veterinary echocardiography. Baltimore: Williams \& Wilkins, 1998. p.151-260.

DÁVILA-ROMÁN, V.G. et al. Transient right but not left ventricular dysfunction after strenuous exercise at high altitude. Journal of the American College of Cardiology, v.30, n.2, p.468-473, 1997. Disponível em: <http:// www.ncbi.nlm.nih.gov/pubmed/9247520>. Acesso em: 20 out. 2010. doi: 9247520 .

DOUGLAS, P.S. et al. Cardiac fatigue after prolonged exercise. Circulation, v.76, p.1206-1213, 1987. Disponível em: <http:/ /circ.ahajournals.org/cgi/content/abstract/76/6/1206 >. Acesso em: 20 out. 2010. doi: 76/6/1206. 
DURANDO, M.M. et al. Right ventricular pressure dynamics during exercise: relationship to stress echocardiography. Equine Veterinary Journal Supplement, v.34, p.472-477, 2002. Disponível em: <http://onlinelibrary.wiley.com/doi/10.1111/ j.2042-3306.2002.tb05468.x/abstract>. Acesso em 20 out. 2010. doi: 10.1111/j.2042-3306.2002.tb05468.x.

FLAMÍNIO, M.J.B.; RUSH, B.R. Fluid and electrolyte balance in endurance horses. Veterinary Clinics of North America Equine Practice, v.14, p.147-158, 1998. Disponível em: $<$ http://www.ncbi.nlm.nih.gov/pubmed/9561693> Acesso em: 20 out. 2010 . doi: 9561693.

GEHLEN, H. et al. Echocardiographic comparison of left ventricular dimensions and function after standardized treadmill exercise in trained and untrained healthy warmblood horses. Equine and Comparative Exercise Physiology, v.3, n.1, p.3-11, 2005. Disponível em: <http://journals.cambridge.org/ action/displayAbstract;jsessionid $=84 \mathrm{C} 291 \mathrm{E} 4364 \mathrm{~F} 5215 \mathrm{~A} 30 \mathrm{CE}$ D84E3B666B 9. tomcat 1 ? fromPage $=$ online $\&$ aid $=793936>$. Acesso em: 20 out. 2010. doi: 793936.

POOLE, D.C.; ERICKSON, H.H. Cardiovascular function and oxygen transport: responses to exercise and training. In:

Equine exercise physiology: the science of exercise in the athletic horse. London: Saunders, 2008. p.221-235.

KALLIOKOSKI, K.K. et al. Myocardial perfusion after marathon running. Scandinavian. Journal of Medicine \& Science in Sports, v.14, n.1, p.208-214, 2004. Disponível em: <http://onlinelibrary.wiley.com/doi/10.1111/j.16000838.2004.00357.x/full>. Acesso em: 20 out. 2010. doi: 10.1111/j.1600-0838.2004.00357.x.

LA GERCHE, A. et al. No evidence of sustained myocardial injury following an Ironman distance triathlon. International Journal of Sports Medicine, v.25, p.45-49, 2004. Disponível em: <https://www.thieme-connect.com/ejournals/abstract/ sportsmed/doi/10.1055/s-2003-45236>. Acesso em: 20 out. 2010. doi: $10.1055 / \mathrm{s}-2003-45236$.

MARR, C.M. et al. Pre-and post exercise echocardiography in horses performing treadmill exercise in cool and hot/humid conditions. Equine Veterinary Journal Supplement, v.30, p.131-136, 1999. Disponível em: <http://onlinelibrary.wiley.com/ doi/10.1111/j.2042-3306.1999.tb05203.x/abstract>. Acesso em: 20 out. 2010. doi: 10.1111/j.2042-3306.1999.tb05203.x.

MICHIMA, L.E.S. et al. Comparative study of echocardiographic dimensions and indices between endurance race horse with good and poor performance, raised in São Paulo State, Brazil. In: CONGRESO PANAMERICANO DE CIENCIAS VETERINARIAS, 2004, Buenos Aires. Abstracts Book... Buenos Aires: Congresos Internacionales, 2004. p.6061.

MICHIMA, L.E.S. Influência do exercício físico prolongado sobre a concentração sérica de troponina I cardíaca e sobre a função cardíaca em cavalos de enduro. 2007. 94f. Tese (Doutorado em Clínica Veterinária) - Curso de Pós-graduação em Medicina Veterinária, Faculdade de Medicina Veterinária e Zootecnia, Universidade de São Paulo, SP.

NAYLOR, J.R. et al. Effects of dehydration on thermoregulatory responses of horse during low-intensity exercise. Journal Applied Physiology, v.75, p.994-1001, 1993. Disponível em: <http://jap.physiology.org/cgi/content/ abstract/75/2/994>. Acesso em: 20 out. 2010. doi: 8750$7587 / 93$.

NEILAN, T.G. et al. Among nonelite participants in the Boston marathon myocardial injury and ventricular dysfunction related to training levels. Circulation, v.114, p.2325-2333, 2006. Disponível em: <http://circ.ahajournals.org/cgi/content/abstract/ 114/22/2325>. Acesso em 20 out. 2010. doi: 10.1161/ CIRCULATIONAHA.106.647461.

REEF, V.B. Echocardiographic examination in the horse: the basics. Compendium on Continuing Education for the Veterinarian Practicing, v.12, n.9, p.312-319, 1990.

RIFAI, N. et al. Cardiac troponin T and I, electrocardiographic wall motion analyses, and ejection fractions in athletes participating in the Hawaii ironman triathlon. American Journal of Cardiology, v.83, p.1085-1089, 1999. Disponível em: <http://www.ajconline.org/article/S0002-9149(99)00020$\mathrm{X} / \mathrm{abstract}>$. Acesso em: 20 out. 2010. doi: S00029149(99)00020-X.

ROSE, R.J. An evaluation of heart rate and respiratory rate recovery for assessment of fitness during endurance rides. In: PERSSON, S.G.B. et al. Equine exercise physiology. Cambridge: Granta Editions, 1983. p.505-509.

SCHEFER, K.D. et al. Quantitative analysis of stress echocardiograms in healthy horses with 2-dimensional (2d) echocardiography, anatomical m-mode, tissue doppler imaging, and $2 \mathrm{~d}$ speckle tracking. Journal of Veterinary Internal Medicine, v.24, p.918-931, 2010. Disponível em: <http:// on linelibrary.wiley.com/doi/ $10.1111 /$ j. 1939 1676.2010.0542.x/abstract>. Acesso em: 20 out. 2010. doi: 10.1111/j.1939-1676.2010.0542.x.

SWENSON, M.J.; REECE, W.O. Dukes: fisiologia dos animais domésticos. Rio de Janeiro: Guanabara Koogan, 1996. 856p.

TEICHOLZ, L.E. et al. Problems in echocardiographic volume determinations: echocardiographic-angiographic correlations in the presence or absence of synergy. American Journal of Cardiology, v.37, p.7-11, 1976.

TRIVAX, J.E. et al. Acute cardiac effects of marathon running. Journal Applied Physiology, v.108, p.1148-1153, 2010. Disponível em: <http://jap.physiology.org/cgi/content/abstract/ 108/5/1148>. Acesso em 20 out. 2010. doi: 10.1152/ japplphysiol.01151.2009 8750-7587/10.

WELSH, R.C. et al. Prolonged strenuous exercise alters the cardiovascular response to dobutamine stimulation in male athletes. Journal of Physiology, v.569, n.1, p.325-330, 2005. Disponível em: <http://onlinelibrary.wiley.com/doi/10.1113/ jphysiol.2005.096412/full>. Acesso em: 20 out. 2010. doi: 10.1113/jphysiol.2005.096412.

YAMAZAKI, H. et al. Left ventricular function after prolonged exercise. Kokyu To Junkan, v.58, p.1241-1245, 1990. Disponível em: <http://www.ncbi.nlm.nih.gov/pubmed/ 2287820 s. Acesso em 20 out. 2010. doi: 2287820. 